\title{
Transient Quantum Coherent Response to a Partially Coherent Radiation Field
}

\author{
Z. S. Sadeq and P. Brumer* \\ Chemical Physics Theory Group, Department of Chemistry \\ and Center for Quantum Information and Quantum Control \\ University of Toronto, Toronto, M5S 3H6, Canadd*
}

(Dated: January 12, 2021)

\begin{abstract}
The response of an arbitrary closed quantum system to a partially coherent electric field is investigated, with a focus on the transient coherences in the system. As a model we examine, both perturbatively and numerically, the coherences induced in a three level $V$ system. Both rapid turn-on and pulsed turn-on effects are investigated. The effect of a long and incoherent pulse is also considered, demonstrating that during the pulse the system shows a coherent response which reduces after the pulse is over. Both the pulsed scenario and the thermally broadened CW case approach a mixed state in the long time limit, with rates dictated by the adjacent level spacings and the coherence time of the light, and via a mechanism that is distinctly difference from traditional decoherence. These two excitation scenarios are also explored for a minimal "toy" model of the electronic levels in pigment protein complex PC645 by both a collisionally broadened CW laser and by a noisy pulse, where unexpectedly long transient coherence times are observed and explained. The significance of environmentally induced decoherence is noted.
\end{abstract}

PACS numbers:

*Electronic address: pbrumer@chem.utoronto.ca 


\section{INTRODUCTION}

Recent advances [1, 2] in coherent femtosecond nonlinear spectroscopy have shown evidence of long-lived coherences in photosynthetic systems. However, in nature biological systems are exposed to incoherent sources of light, such as sunlight, as opposed to coherent femtosecond sources. Therefore, an understanding of excitation under incoherent conditions is crucial. In such cases, the long time result of incoherent irradiation, using both quantum and classical light, leads to a mixed state of the system [3, 4], a result discussed further in the literature in the context of photosynthetic light harvesting complexes [5-7]. However, work has been done, for example, on incoherent or noisy perturbations in quantum optics where some have found that incoherent or noisy electric fields can generate transient coherences in three level atoms [8 10]. Hence, understanding the approach to the mixed state, and its dependence on system parameters is important, and is the subject of this paper.

Here, transient excited state coherences manifest upon the perturbative sudden turn-on irradiation of an isolated quantum system are discussed and examined in a V level system. These coherences are shown to become negligible in the long time limit relative to the populations so that the system approaches, in this way, a mixed state. The dynamics of

long and noisy pulses incident on generic systems is also investigated. These pulses induce coherences on a time scale dependent on the excited state period and the pulse duration. However, after the pulse is over these coherences become damped and irrelevant relative to the magnitude of populations. In both cases, the magnitude of the spacing between the excited levels is shown to play an important role. Further, in both cases, the approach to the mixed state is dramatically different than that associated with decoherence experienced by a prepared superposition state that is in contact with a thermal bath (see, e.g., [11, 12]).

We consider the response of a system to two sample sources of incoherent light. The first models incoherent light corresponding to a collisionally broadened CW source [13] that is turned on abruptly. This incoherent light source is characterized by a two time correlation function [3]:

$$
\left\langle\varepsilon\left(t^{\prime}\right) \varepsilon^{*}\left(t^{\prime \prime}\right)\right\rangle=\varepsilon_{0}^{2} e^{-i \omega_{0}\left(t^{\prime}-t^{\prime \prime}\right)} e^{-\frac{\left|t^{\prime}-t^{\prime \prime}\right|}{\tau_{d}}}
$$

where $\omega_{0}$ is the frequency center of the radiation, and $\varepsilon_{0}^{2}$ is the field intensity. The coherence time of this radiation is given by $\tau_{d}=\hbar / k T$ where $k$ is Boltzmann's constant and $T$ is temperature [14, 15]. Hence, a room temperature source at $T=300 \mathrm{~K}$ gives $\tau_{d} \simeq 25$ fs 
whereas a source with $T=5800 \mathrm{~K}$, gives $\tau_{d} \simeq 1.32 \mathrm{fs}$. This corresponds to a classical model of incoherent light; the full quantum version has qualitatively similar behavior to the correlation function in Eq. (1) at these temperatures [13 15].

A second source examined below is a noisy pulse, a Gaussian pulse with a phase jitter designed to model an incoherent light pulse, given by [16]:

$$
\left\langle\varepsilon\left(t^{\prime}\right) \varepsilon^{*}\left(t^{\prime \prime}\right)\right\rangle=\varepsilon_{0}^{2} e^{-\frac{\left(t^{\prime}-t_{m}\right)^{2}}{\tau_{p}^{2}}} e^{-\frac{\left(t^{\prime \prime}-t_{m}\right)^{2}}{\tau_{p}^{2}}} e^{i \omega_{0}\left(t^{\prime \prime}-t^{\prime}\right)} e^{-\frac{\left(t^{\prime}-t^{\prime \prime}\right)^{2}}{2 \tau_{d}^{2}}}
$$

Natural processes experiencing incoherent light can be modeled using Eq. (2) with the pulse duration $\tau_{p}$ being much larger than the coherence parameter, $\tau_{d}$. In this limit Eq. (2) behaves like Eq. (1), but with a smooth turn-on of the field. The attractiveness of using a long incoherent pulse is two fold: (1) it has no sudden "turn-on effect" that leads to artificial initial coherence, and (2) it offers a reasonable long time result which models radiation induced decoherence [16]. In addition, in the pulsed scenario, there are three time scales of interest: $\tau_{d}$ the pulse coherence time, $\tau_{p}$ the pulse duration, and $\tau_{c}$ the characteristic time scale of the system, usually equal to the inverse of the level spacing. In the simulations below we assume that the pulse duration $\tau_{p}$ is much longer than both $\tau_{d}$ and $\tau_{c}$. In addition since, in a natural environment, the system is initially in a stationary mixed state, we consider the light to be incident on a single molecular eigenstate, i.e. a representative element of the ensemble. Finally, note that both Eqs. (1) and (2) are broadened about a single frequency $\omega_{0}$. Typical natural light, however, is a mixture of different $\omega_{0}$ values.

Below we consider both analytic first order perturbation theory results as well as numerical results for these cases. In the numerical implementation, the correlation functions [Eqs. (1) and (2)] are produced as averages over many individual realizations built from a Wiener process (see Appendix A), i.e., electric fields $\varepsilon(t)$ with suitable distribution of jumps in phase [17]. Specifically, the collisionally broadened CW source is reproduced by perturbing a classical oscillator's frequency with Wiener noise. The noisy pulse is generated in a similar fashion, except that a Gaussian envelope is placed over the noisy oscillator.

A remark is in order with respect to this approach. Physically, the meaningful quantity is the molecular density matrix that arises from the effect of the ensemble of electric fields that satisfy the statistics in Eqs. (1) and (2). Such electric field statistics can arise from a wide variety of different types of realizations. For example, it has been suggested that 
coherent fs pulses may be a meaningful basis from which to build such realizations [18]. To do so requires that the pulses be added together incoherently casting doubt on the relevance of the coherence observed in any single realization. In addition, such a fs basis, unlike our choice of phase interruptions to a CW source, has no physical justification.

The paper is organized as follows. Section II considers the short time response of a closed quantum system, both perturbatively and numerically, to a collisionally broadened CW source, Eq. (1) after sudden turn-on. Section III deals with the case of long incoherent pulses, Eq. (2) incident on a model molecular system, both perturbatively and numerically. A toy model of the electronic energy levels of the pigment-protein complex PC645 is used in both sections as an interesting example. The paper is summarized in Sec. IV.

\section{SHORT TIME RESPONSE OF AN ENERGY LEVEL TO INCOHERENT LIGHT}

The effect of CW incoherent light incident on a molecule in an energy eigenstate and starting in the infinite past, is treated in detail in Refs. [3, 4]. There, the effect was to produce, in the long time limit, a mixture of stationary system energy eigenstates. Absent from this treatment was the dynamics by which this came about, which is the subject of this section. Note that given the weakness of natural light (such as sunlight) a first-order perturbative treatment is applicable.

\section{A. Perturbative Treatment (Analytic)}

The total Hamiltonian of the system and radiation is given by $H=H_{0}-\mu \varepsilon(t)$, where $\mu$ is the dipole operator and $\varepsilon(t)$ is the electric field. Using the standard dipole approximation and treating the light-molecule interaction to first order gives the following expression [3] for the excited state density matrix:

$$
\rho_{e}=\sum_{i, j} c_{i} c_{j}^{*}|i\rangle\langle j| e^{-i \omega_{i j} t} \int_{t_{0}}^{t} d t^{\prime} e^{i \omega_{i g} t^{\prime}} \int_{t_{0}}^{t} d t^{\prime \prime} e^{-i \omega_{j g} t^{\prime \prime}}\left\langle\varepsilon\left(t^{\prime}\right) \varepsilon^{*}\left(t^{\prime \prime}\right)\right\rangle .
$$

Here, the time independent coefficients $c_{i}, c_{j}^{*}$ are given by $c_{i}=\varepsilon_{0}\langle i|\mu| g\rangle / i \hbar$ where the $|i\rangle$ are the energy eigenstates of the system at energy $E_{i}$ and $\omega_{j g}=\left(E_{j}-E_{g}\right) / \hbar$ is the frequency difference between the $j$ th state and the ground state. The initial condition has the system 
in the ground state, i.e., $\rho(0)=|g\rangle\langle g|$. Equation (3) has been obtained assuming that the ensemble average over the product of the electric fields and the time integrals commute. The validity of this assumption is confirmed numerically in Sec. IIB.

For the collisionally broadened CW source, we insert Eq. (1) for $\left\langle\varepsilon\left(t^{\prime}\right) \varepsilon^{*}\left(t^{\prime \prime}\right)\right\rangle$ into Eq. (3) and compute the two time integrals. The integral can be written as four distinct terms, so that the excited state density matrix is:

$$
\begin{gathered}
\rho_{e}=\sum_{i, j} c_{i} c_{j}^{*}\left|E_{i}\right\rangle\left\langle E_{j}\right|\left(\eta_{L T}^{i j}+\eta_{1}(i j)+\eta_{2}(i j)+\eta_{3}(i j)\right) \\
\eta_{L T}^{i j}=i \mathcal{U}\left(\omega_{j g}\right) \frac{e^{-i \omega_{i j} t}-1}{\omega_{i j}} \\
\eta_{1}(i j)=-\frac{\mathcal{U}\left(\omega_{j g}\right)}{2} \frac{e^{t\left(i \delta_{j}-\frac{1}{\tau_{d}}\right)}-e^{-i \omega_{i j} t}}{i \delta_{i}-\frac{1}{\tau_{d}}} \\
\eta_{2}(i j)=-\frac{\mathcal{U}\left(\omega_{j g}\right)}{2}\left(\frac{1-e^{-i\left(\delta_{j}+\omega_{i j}\right) t} e^{-\frac{t}{\tau_{d}}}}{\frac{1}{\tau_{d}}+i \delta_{i}}\right) \\
\eta_{3}(i j)=i R\left(\delta_{j}\right)\left(\frac{1-e^{-i\left(\delta_{j}+\omega_{i j}\right) t} e^{-\frac{t}{\tau_{d}}}}{\frac{1}{\tau_{d}}+i \delta_{i}}-\frac{e^{i \delta_{j} t} e^{-\frac{t}{\tau_{d}}}-e^{-i \omega_{i j} t}}{i \delta_{i}-\frac{1}{\tau_{d}}}\right)
\end{gathered}
$$

For simplicity, we have set $t_{0}=0$; averaging over $t_{0}$ is discussed in Appendix B. Here, $\delta_{k}=\omega_{k g}-\omega_{0}$ gives the detuning of the $|g\rangle \rightarrow|k\rangle$ transition frequency from the central laser frequency, and $R\left(\delta_{j}\right)=\delta_{j} /\left(\frac{1}{\tau_{d}^{2}}+\delta_{j}^{2}\right)$ is a Lorentzian that is dependent on the detuning and on the coherence parameter $\tau_{d}$.

The first term, $\eta_{L T}^{i j}$, tends to dominate at long times and the $\eta_{k}(i j)$ contain transient terms that decay on the field coherence time, $\tau_{d}$. The terms $\eta_{L T}^{i j}, \eta_{1}, \eta_{2}$ are preceded by a Lorentzian $\mathcal{U}(\omega)$ that is characteristic of the Wiener process underlying the electric field statistics:

$$
\mathcal{U}(\omega)=\frac{2 \tau_{d}}{1+\tau_{d}^{2}\left(\omega-\omega_{0}\right)^{2}}
$$

In the limit of $\tau_{d} \rightarrow \infty, \mathcal{U}(\omega)$ approaches $\delta\left(\omega-\omega_{0}\right)$, thus converging to the Fourier transform of a conventional CW laser.

As shown below, $\eta_{L T}^{i i}$ is a term that contributes significantly to the populations as time increases. Specifically, in the limit of $\omega_{i j} \rightarrow 0$,

$$
\eta_{L T}^{i i}=\mathcal{U}\left(\omega_{i g}\right) t
$$


Alternatively, this result can be obtained by solving Eq. (3) in the limit of $j \rightarrow i$.

By examining Eqs. (4) - (8) after a time, $t \gg \tau_{d}$, one sees that the contributions from the $\eta_{k}, k=1,2,3$ are either negligible or oscillate on a time scale of the inverse of the level spacing, similar to the $\eta_{L T}^{i j}$ term. As the system evolves, the populations grow at a constant rate, while the amplitude of the coherence become fixed (but non-zero) after time $\tau_{d}$. This implies that at some long time $\left(t \gg \tau_{d}\right)$, the populations become extremely large relative to coherences, i.e. it is in this way that the system reaches a mixed state. It also indicates that at long times the off-diagonal elements $\rho_{i j}$ of $\rho_{e}$ are usually small but nonzero. Indeed, since the magnitude of $\eta_{L T}^{i_{j}}$ is inversely proportional to $\omega_{i j}$, nearby levels can display large coherences. These off-diagonal elements are a direct reflection of the open nature of the quantum system; that the system is coupled to the radiative bath, resulting in a system-bath coupling that leads to a non-diagonal system density matrix in the original system energy basis [6]. This is an example of "canonical-nontypicality" and is of particular interest in topics like one-photon phase control [19]

The expression for populations is in agreement with that in Ref. [5]. However, they are in contrast with our earlier results of Ref. [3] that give the density matrix as diagonal in the energy eigenbasis. The difference results from the choice, in Ref. [3], of a turn-on in the infinite past and of a continuous energy distribution. Nonetheless, the qualitative result here is the same as that in Ref. [3, i.e., the system is found in a mixed state at long times.

Issues of the introduction of a specific laser turn-on time are discussed in Appendix $\mathrm{B}$ where averaging over turn-on time $t_{0}$ is shown not to eliminate the small coherences encountered in this approach. In addition, Appendix B discusses conditions under which the long time $\rho_{i j}$ are maximal (but still vanishingly small compared to the populations).

These results indicate (1) the nature of the closed system transient coherent response to the sudden turn-on of an incoherent field, (2) the linearity of the population growth due to the diffusion process underlying the radiation field, (3) that the coherences so induced do survive as time evolves, but (4) that populations become much larger than the coherences, so that the system effectively approaches a mixed state.

Sample computations quantifying these results, and allowing for an analysis of the dependence on system properties, are provided below. 


\section{B. Perturbative Treatment (Numerical)}

Although the perturbation associated with natural sunlight is weak, and hence Eqs. (4) - (8) are expected to give virtually exact results, numerical studies are necessary for the pulsed case considered below (Sect. III) and for confirmation of the validity of ordering of the time integral and ensemble averaging assumed above. Below, the perturbation theory results are compared to numerical results obtained by solving the von Neumann equation of a $V$ level system:

$$
\frac{d \rho}{d t}=\frac{i}{\hbar}[\rho, H]
$$

where the Hamiltonian is given by:

$$
H=\left(\begin{array}{ccc}
\omega_{g} & -\mu \varepsilon(t) & -\mu \varepsilon(t) \\
-\mu \varepsilon^{*}(t) & \omega_{1} & 0 \\
-\mu \varepsilon^{*}(t) & 0 & \omega_{2}
\end{array}\right)
$$

and where the star denotes the complex conjugate.

We generate collisionally broadened CW sources by producing a set of fields $\{\varepsilon(t)\}$, which obeys the statistics in Eq. (1). This was done by changing the phase at random times with the interruption times selected from a Wiener distribution, and phase changes chosen from a uniform distribution. Details of this algorithm are in Appendix A.

A model $V$ level system, illustrated in Fig. 1, with initial population in the ground state was subjected to a set of fields $\{\varepsilon(t)\}$. An average over the set of individual realizations of $\varepsilon(t)$ gives the resultant $\rho$. To compare to the perturbative result obtained in Sec. IIA we focus on the excited populations $\rho_{22}, \rho_{33}$, and coherences between excited states $\rho_{23}$.

Figure 2 shows the perturbative vs. numerical calculations for the excited state populations and coherences for a fixed value of $\tau_{d}=120$ fs. This $\tau_{d}$ value, which is two orders of magnitude larger than that of solar radiation, is examined for convenience only. The frequency of radiation used is chosen to be $\omega_{0}=\left(\omega_{31}+\omega_{21}\right) / 2$ so as to excite both transitions equally and here and below, unless otherwise indicated, $\mu \varepsilon_{0} / \hbar=1 \mathrm{THz}$. Transient field induced terms are clear at short times, but after $t \gg \tau_{d}$ the system oscillates at the

level spacing, here chosen to be $\tau_{c}=\frac{2 \pi}{\omega_{32}} \simeq 60$ fs. The ensemble averaged excited state populations show linear growth, agreeing with the dynamics predicted by the perturbative 
result derived earlier. The perturbative and numerical solutions show excellent agreement for coherences while the numerical solution has a slightly smaller slope to that of the perturbative result for populations. This is due to the inability to generate the exact correlation function accurately, as discussed in Appendix A.

To examine the coherence of the created excited state, we define

$$
\mathcal{C} \equiv\left|\left\langle\rho_{23}\right\rangle\right| /\left(\left\langle\rho_{33}\right\rangle+\left\langle\rho_{22}\right\rangle\right)
$$

as a measure of the mixed state character; here, $\mathcal{C}=0.5$ corresponds to that of an equal coherent superposition of two states. We plot this quantity, as well as the purity for the entire system $\operatorname{Tr}\left[\rho^{2}\right]$, in Figs. 3 and 4 for several values of $\tau_{c}$ and $\tau_{d}$. To complement our measure $\mathcal{C}$ we also plot the full system purity $\operatorname{Tr}\left(\rho^{2}\right)$ in Fig. 4 and the excited state purity $\operatorname{Tr}\left(\rho_{e}^{2}\right) /\left(\operatorname{Tr}^{2}\left(\rho_{e}\right)\right)$ in Fig. 5 .

As seen in Figs. 2 and as manifest in Fig. 3, as the system evolves under incoherent excitation, coherences stay the same order of magnitude while the populations increase. In the long time limit (not shown), we recover the limit in Ref. [3] insofar as the state populations become overwhelmingly larger than the coherences between them, i.e., $\mathcal{C} \rightarrow 0$ in the limit of $t \rightarrow \infty$. This time becomes longer with increasing $\tau_{d}$ but is, of course, infinitesimally small in cases of natural light, which illuminates for macroscopic time scales. Note that both $\mathcal{C}$ and the excited state purity are found to behave similarly and that the coherence is seen to survive longer for larger $\tau_{d}$ and larger $\tau_{c}$. The curves show decaying oscillations at a frequency $2 \pi / \tau_{c}$.

Examining the plot of $\operatorname{Tr}\left[\rho^{2}\right]$ in Fig. 4 it is evident that the total purity decreases with time, where the smaller $\tau_{c}$ is relative to $\tau_{d}$, the less pure the state becomes. This effect is, however, mainly due to increased population in the excited state levels, determined by $\mathcal{U}(\omega)$ [see Eq. (9)]. For example, when $\omega_{0}$ is in the center of the levels, $\mathcal{U}=8 \tau_{d} /\left(4+\tau_{d}^{2} \omega_{32}^{2}\right)$. By plotting this as function of $\tau_{d}$ and $\omega_{32}$ (Fig. 6) it is clear that as the level spacing becomes larger, the rate of pumping decreases, and vice versa.

\section{Toy PC645 in a Collisionally Broadened CW Source}

Intense interest has surrounded the observation of quantum coherence in photosynthetic pigment protein complexes [20], observed with coherent laser light. However, under natural 
conditions, these protein complexes are irradiated with sunlight. Here we utilize $V$ level system as a toy model for the electronic energy levels of PC645 and focus is on obtaining qualitative insight into the time scales for coherences due to coupling to the chaotic light. The upper levels $|2\rangle$ and $|3\rangle$ represent $\left|D B V^{-}\right\rangle$and $\left|D B V^{+}\right\rangle$electronic states that are excited experimentally [20, 21]. This model PC645 level structure is irradiated with the collisionally broadened CW source using the perturbative approach described above. The laser frequency $\omega_{0}$ was chosen to be in the center of the two levels so as to excite both transitions equally and the coherence time $\tau_{d}=1.32 \mathrm{fs}$, is that of sunlight. Electric field intensities are taken from literature values of the intensity of the sun at midday [22]. Site energies and dipole moments given in Appendix D were taken from literature [21]. The perturbative calculation for the coherences and populations of the excited states, where the field is turned on at $t_{0}=0$, are presented in Figs. 7 and 8 .

Once again from Figs. 7 and 8 it is evident that there is a transient coherent response associated with the sudden turn-on that becomes an increasingly smaller fraction of the excited state population, as evidenced by the measure $\mathcal{C}$. For example, for $t>500$ fs, $\mathcal{C}<0.05$, i.e., the coherences are already very small relative to population. Since the population growth is linear in time, $\mathcal{C}$ decays to zero as $\left|\sin \left(\omega_{23} t\right)\right| /(2 t)$. Note, similar to the computations above, the timescale over which the coherences are a significant fraction of the populations is far larger than $\tau_{d}$. This is consistent with our observation that this timescale increases as $1 / \tau_{c}$.

This calculation was done on an isolated quantum system, without the inclusion of external degrees of freedom corresponding to the local vibrations and the protein environment. Depending on the parameters of the bath, the decoherence associated with system-bath interactions could speed up the decoherence experienced by interacting with light or have negligible impact. Since excitation from pigment protein complexes need tens of picoseconds to arrive at the reaction center, it is clear, even from this toy model, that it cannot do so via pure electronic coherent dynamics.

These computations assume the sudden turn-on of the incoherent light, which induces strong coherences. The issue of a slow turn-on is addressed in the following section. 


\section{RESPONSE OF AN ISOLATED QUANTUM SYSTEM TO LONG INCOHER- ENT PULSES}

A potential issue associated with using the collisionally broadened CW source discussed above is the "sudden turn-on effect" which will induce artificial coherences. This issue can be examined by using a "noisy pulsed source" [16], with a two time correlation function given in Eq. (2). However, using the perturbative approach outlined in Sec. IIA only gives analytic results only for times $t \gg \tau_{p}$, where $\tau_{p}$ is the pulse duration. Specifically, inserting Eq. (2) into Eq. (3) and setting $t_{0} \rightarrow-\infty$ and $t \rightarrow \infty$ gives

$$
\begin{gathered}
\rho_{e}\left(t \gg \tau_{p}\right)=\sum_{i, j} c_{i} c_{j}^{*}\left|E_{i}\right\rangle\left\langle E_{j}\right| e^{-i \omega_{i j} t} \eta_{P}^{i j} \\
\eta_{p}^{i j}=\tau_{p} T e^{i t_{m} \omega_{i j}} \exp \left(-\frac{\tau_{p}^{2} \omega_{i j}^{2}}{8}\right) \exp \left(-\frac{T^{2}}{8}\left(\delta_{i}+\delta_{j}\right)^{2}\right)
\end{gathered}
$$

where $T=\tau_{p} \tau_{d} / \sqrt{\tau_{p}^{2}+\tau_{d}^{2}}$

Note that in the limit of $\tau_{p} \gg \tau_{d}, T \rightarrow \tau_{d}$. Further note that, unlike the sudden turn-on case, averaging over pulse centers $t_{m}$ would cause coherences to vanish. Using Eq. (14) gives a general form for $\mathcal{C}$ between states $i$ and $j$ of

$$
\mathcal{C}=\frac{\exp \left(-\tau_{p}^{2} \omega_{i j}^{2} / 8\right) \exp \left(-T^{2}\left(\delta_{i}+\delta_{j}\right)^{2} / 8\right)}{\exp \left(-T^{2} \delta_{i}^{2} / 2\right)+\exp \left(-T^{2} \delta_{j}^{2} / 2\right)}
$$

For the specific case where the $\omega_{0}$ lies between the two eigenvalues, $\delta_{i}=-\delta_{j}=\omega_{i j} / 2$. Hence, in this case,

$$
\mathcal{C}=\frac{1}{2} \exp \left(-\tau_{p}^{2} \omega_{i j}^{2} / 8\right) \exp \left(T^{2} \delta_{i}^{2} / 2\right)
$$

which, if $\tau_{p} \gg \tau_{d}$, becomes

$$
\mathcal{C}=\frac{1}{2} \exp \left[\left(\tau_{d}^{2}-\tau_{p}^{2}\right) \omega_{i j}^{2} / 8\right]=\frac{1}{2} \mathrm{e}^{-\tau_{p}^{2} \omega_{i j}^{2} / 8}
$$

The latter expressions assume that the dipole moment of the excited states are equal. We have performed numerical calculations on such systems and these results, which allow for an appreciation of the coherences generated during the pulse, are presented in Fig. 9 where $\tau_{p}=$ 1 ps. During the pulse, the excited state coherences can be seen to be a significant fraction of the excited state population. However, after the pulse is over, these coherences become a negligible fraction of the populations. Note that as $\tau_{c}$ becomes larger, the coherent response 
during the pulse and post-pulse becomes larger. For $\tau_{c}$ approaching $\tau_{p}$ the decoherence is significantly less than when $\tau_{c}$ is considerably smaller than $\tau_{p}$. Indeed, for $\tau_{p}=1 \mathrm{ps}$ and $\tau_{c}=500 \mathrm{fs} \mathcal{C}$ is seen to be constant at 0.24 after the pulse is over, since both populations and coherences are constant after that time.

These results are in contrast to those of the collisionally broadened CW source insofar as the time $\tau_{d}$ plays little role. Rather, from the perturbative expression [Eq. (18)] $\mathcal{C}$ is seen to be dependent only on the ratio of pulse duration and excited state splitting. Hence, even for incoherent sources with very small $\tau_{d}$ the pulse can create a partially coherent superposition between excited states. This is particularly the case for levels that are very closely spaced, e.g., vibrational levels in small molecules. This is consistent with observations made in Ref. [3], where note was made of the fact that such coherence is generated because the envelope of the pulse is, itself, smooth.

\section{A. Irradiation of Toy PC645 Using Noisy Pulses}

As a demonstration of the response of electronic levels using typical biological system parameters, consider the irradiation of a toy PC645 molecule with a noisy pulse. The model for PC645 is the same as in Sec. IIC. The post pulse expression for $\mathcal{C}$, plotted as a function of pulse duration, is shown in Fig. 10 where $\tau_{d}<\tau_{p}$ is not assumed. For short pulses (sub 100 fs) partially coherent excited states can be created, but for pulses longer than 100 fs the coherence is negligible. Hence, excitation of electronic superpositions using incoherent sunlight of macroscopic time scale duration, is not expected.

\section{SUMMARY}

Understanding the response of molecular systems to incoherent light, such as sunlight, is vital in efforts to advance studies of natural light harvesting processes, photovoltaics, etc. Previous work [3 5] showed that the density matrix of the molecule in the long time limit was that of a mixed state, with no evident time dependent quantum coherence.

This paper has carefully examined the nature of the transient coherences associated with molecular excitation with incoherent light for two paradigmatic cases, the sudden turn-on of collisionally broadened CW light, and the excitation by a Gaussian pulse with phase jitter. 
The role of the decoherence time of the radiation $\tau_{d}$, the pulse duration $\tau_{p}$, and the system timescale as measured by the inverse of the energy level spacing $\tau_{c}$, were examined.

For the case of sudden turn-on of collisionally broadened light, time dependent coherences, although persistent, were found to become insignificant relative to the populations as time involved. Both larger $\tau_{d}$. and larger $\tau_{c}$ enhanced the timescales of these coherences. Averaging over the start time of the sudden turn-on resulted in the survival of time independent (stationary) coherences that were larger for larger $\tau_{c}$.

In the pulsed case, the ratio of the coherences to the population is, for times long after the pulse is over, and where $\tau_{p}>>\tau_{d}$, heavily determined by the ratio of the pulse duration to the level spacing, i.e. $\tau_{p} / \tau_{c}$. Specifically, the larger this ratio, the greater the decoherence. Qualitatively, the $\tau_{p}$ dependence arises from the fact that the longer the pulse is on, the larger the population in the excited levels. As noted earlier in [3] molecules irradiated by pulses with smooth envelopes, even if there are phase jumps, pick up coherence from the smooth pulse envelope. Hence, results are expected to differ for models that employ, e.g., erratic pulse amplitudes.

Both cases show that the mixed state comes about in a fashion distinctly different than that in scenarios typically used to explore decoherence (see, e.g., Refs. [11, 12]). In those cases the effect of an environment on an initial superposition state, with no external driving field, shows a characteristic decay of coherences, i.e. the decay of off-diagonal $\rho_{i j}$ of the system density matrix. In the case studied here, these off-diagonal elements do not decay, but the ratio of these elements to the populations decrease as time goes on, since the populations increase due to the external driving field. Interestingly, despite these differences, the two scenarios do share the common feature, i.e. that the approach to a mixed state is slower for smaller level spacings, i.e., large $\tau_{c}$. Hence, extended studies on incoherent light excitation in dense vibronic manifolds in large molecules is certainly well motivated, and is underway[23].

The fact that, in both sudden turn-on and pulsed cases, larger $\tau_{c}$ enhances coherence times indicates the need to consider decoherence effects of the environment, if the system is open. Such environmental decoherence effects may well serve as the dominant decoherence effect (as opposed to the effect of the incoherent light) for systems with large $\tau_{c}$, a resultant consistent with some earlier considerations [6] in a different context. Further studies of this type on realistic open atomic and molecular systems are in progress. 


\section{Acknowledgments}

ZS thanks OGSST for funding, and Dr. L. Pachon, Y. Khan and R. Dinshaw for edifying discussions. PB thanks Dr. T. Tscherbul for insightful remarks. This work was supported by the Air Force Office of Scientific Research under Contract No. FA9550-13-1-0005 


\section{Appendix A: Numerical Reproduction of Incoherent Light}

\section{Collisionally Broadened CW Laser}

The approach outlined in ref [13, 17] is used to model the incoherent light. In this model the light has a central frequency $\omega_{0}$ and a time dependent phase $\phi(t)$ that abruptly changes $b$ times. The change in phase is taken from a uniform distribution. The times at which these collisions occur, $\left\{t_{j}\right\}$ are governed by a Wiener process $W(t)$ with a distribution center of zero and a drift coefficient $(D)$ that is given by some scaling factor multiplied by the coherence time of the radiation $\tau_{d}$. From this process, a phase interrupted harmonic drive is generated. Each realization has $b$ collisions between the initial time $t_{i}$ to final time $t_{f}$. Several thousand of these realizations are generated, creating a set of electric fields $\{\varepsilon(t)\}$, from which the two time correlation function is computed. The real component of an ith realization is given by:

$$
\begin{aligned}
& \operatorname{Re}\left(\varepsilon_{i}\right)(t) \propto \cos \left(\omega_{0} t+\phi_{i}(t)\right) \\
& \phi_{i}(t)=\phi_{0} \theta\left(-t-t_{i}\right)+\prod_{k} \theta\left(t-t_{k}\right) \phi_{k} \theta\left(-t+t_{k+1}\right)+\phi_{f} \theta\left(t-t_{f}\right)
\end{aligned}
$$

Here, $\theta(t)$ is the Heaviside theta function, $\phi_{m}$ are the phase changes and the set $\left\{t_{j}\right\}$ are the phase interruption times. For the case of $\tau_{d}=120 \mathrm{fs}, b$ is chosen to be a random number

from a uniform distribution between 10 and 12. For different values of $\tau_{d}$ one has to adjust the drift coefficient $D$ and the number of collision events $b$ to reach agreement with the exact correlation function.

Results of this numerical method are plotted in Fig. 11 and compared to the exact expression [Eq. (1)]. It is obvious that, although the numerical method can reproduce most of the true correlation function, it still generates some error. It should be noted that finding the correct parameters ( $b$ and $D$ ) to fit the exact correlation function numerically was non-trivial and highly dependent on the radiation coherence time $\tau_{d}$.

\section{Noisy Pulsed Light}

Generating noisy pulses is similar to that outlined above except that a Gaussian envelope is imposed on incoherent light. 


$$
\begin{aligned}
& \operatorname{Re}\left(\varepsilon_{i}\right)(t) \propto p(t) \cos \left(\omega_{0} t+\phi_{i}(t)\right) \\
& p(t)=\left(\frac{2}{\pi \tau_{p}}\right)^{1 / 4} e^{-\frac{\left(t-t_{m}\right)^{2}}{\tau_{p}}}
\end{aligned}
$$

Here $\tau_{p}$ is the pulse duration and $t_{m}$ is the pulse center. The results of this procedure are shown in Fig. 12 where the numerical and exact correlation functions are compared. It is clear that noisy pulsed source is sufficiently well reproduced by the numerical procedure.

\section{Appendix B: Transient Response as a Function of Turn-on Time for a Wiener CW}

\section{Source}

The treatment in Eq. (3) assumes a sudden turn-on. Of interest is the effect of averaging over the turn-on, which may well occur for an ensemble. To this end, consider an arbitrary turn-on time, $\tau_{0}$

$$
\begin{aligned}
\rho_{e} & =\sum_{i, j} c_{i} c_{j}^{*}\left|E_{i}\right\rangle\left\langle E_{j}\right| e^{-i \omega_{i j} t} \int_{t_{0}}^{t} d t^{\prime} e^{i \omega_{i g} t^{\prime}} \int_{t_{0}}^{t} d t^{\prime \prime} e^{-i \omega_{j g} t^{\prime \prime}}\left\langle\varepsilon\left(t^{\prime}\right) \varepsilon^{*}\left(t^{\prime \prime}\right)\right\rangle \\
& =\sum_{i, j} c_{i} c_{j}^{*}\left|E_{i}\right\rangle\left\langle E_{j}\right| \Upsilon
\end{aligned}
$$

with $\Upsilon=\Upsilon_{1}+\Upsilon_{2}+\Upsilon_{3}$, where

$$
\begin{gathered}
\Upsilon_{1}=\frac{2 i \tau_{d}\left(1-e^{-i \omega_{i j}\left(t-t_{0}\right)}\right)}{\omega_{j i}\left(1+\tau_{d}^{2} \delta_{j}^{2}\right)} \\
\Upsilon_{2}=\frac{\tau_{d}^{2}\left(1-e^{-\frac{\left(t-t_{0}\right)}{\tau_{d}}} e^{-i \delta_{i}\left(t-t_{0}\right)}\right)}{\left(-i+\tau_{d} \delta_{j}\right)\left(-i+\tau_{d} \delta_{i}\right)} \\
\Upsilon_{3}=\frac{\tau_{d}^{2}\left(e^{-i \omega_{i j}\left(t-t_{0}\right)}-e^{-\frac{\left(t-t_{0}\right)}{\tau_{d}}} e^{i \delta_{j}\left(t-t_{0}\right)}\right)}{\left(i+\tau_{d} \delta_{j}\right)\left(i+\tau_{d} \delta_{i}\right)}
\end{gathered}
$$

Consider then an ensemble average over $t_{0}$, where the distribution of start times is assumed uniform, and the average is taken over some time $\left[0, \frac{2 \pi}{\omega_{i j}}\right]$. The terms that are proportional to $e^{-i \omega_{i j} t_{0}}$ go to zero under this average while other terms are weighted by $e^{-\frac{t}{\tau_{d}}}$ and decay on a time scale associated with $\tau_{d}$. Averaging over these terms and neglecting terms that decay for $t \gg \tau_{d}$ gives 


$$
\left\langle\rho_{e}^{i j}\right\rangle \propto \frac{2 i \tau_{d}}{\omega_{j i}\left(1+\tau_{d}^{2} \delta_{j}^{2}\right)}+\frac{\tau_{d}^{2}}{\left(-i+\tau_{d} \delta_{j}\right)\left(-i+\tau_{d} \delta_{i}\right)}
$$

That is, the coherences are found to approach a nonzero value which is a function of the level spacing, radiation coherence time and laser detuning.

\section{Maximizing Stationary Coherences}

Stationary coherences of the type seen in Eq. (B6) are of interest in a number of contexts, e.g., one-photon phase control [19]. Maximizing this term is hence of interest. For convenience, define Eq. (B6) as $F$.

$$
F=\frac{2 i \tau_{d}}{\omega_{j i}\left(1+\tau_{d}^{2} \delta_{j}^{2}\right)}+\frac{\tau_{d}^{2}}{\left(-i+\tau_{d} \delta_{j}\right)\left(-i+\tau_{d} \delta_{i}\right)}
$$

with

$$
|F|^{2}=\frac{\tau_{d}^{2}\left(4+\omega_{j i}^{2} \tau_{d}^{2}\right)}{\omega_{j i}^{2}\left(1+\delta_{i}^{2} \tau_{d}^{2}\right)\left(1+\delta_{j}^{2} \tau_{d}^{2}\right)}
$$

The extrema of Eq. (B8) are then found with respect to $\tau_{d}$ and $\omega_{0}$. The only physical extrema for $\tau_{d}$ is $\tau_{d}=0$ which minimizes coherences. With respect to the laser frequency, the only physical extrema is $\omega_{0}=\left(\omega_{i g}+\omega_{j g}\right) / 2$. In this case, $|F|^{2}$ becomes:

$$
|F|^{2}=\frac{16 \tau_{d}^{2}}{\omega_{j i}^{2}\left(4+\tau_{d}^{2} \omega_{j i}^{2}\right)}
$$

Hence, as the excited state splitting decreases, this long-time coherence becomes much larger.

As an example, using the parameters of PC645 (listed in Appendix D), we plot $|F|^{2}$ in Fig. 13. As the coherence time is increased, it is clear that this stationary coherence eventually saturates. In the large coherence time and with $\omega_{0}=\left(\omega_{i g}+\omega_{j g}\right) / 2$, this stationary coherence becomes:

$$
|F|^{2}=\frac{16}{\omega_{j i}^{4}}
$$

\section{Appendix C: White Noise Perturbative Result}

Most work on incoherent excitation has utilized white noise incident on model systems [9, 10]. White noise is a mathematical construct with no real physical analog. We approach 
this problem from the perturbative approach used in Eq. (3). White noise is given by the following correlation function [9]:

$$
\left\langle\varepsilon\left(t^{\prime}\right) \varepsilon^{*}\left(t^{\prime \prime}\right)\right\rangle=\mathcal{R} \delta\left(t^{\prime}-t^{\prime \prime}\right)
$$

The parameter $\mathcal{R}$ represents the pump power of the source. The Fourier transform of white noise contains all frequencies uniformly, exciting all states.

We use the same approach as outlined by Eq. (3) and substitute Eq. (C1) for the correlation function of the radiation field. Solving for the populations of the excited states we get:

$$
\rho_{e}^{i i}=\frac{\left|c_{i}\right|^{2}}{\hbar^{2}} \mathcal{R} t
$$

Solving for coherences we get:

$$
\rho_{e}^{i j}=\frac{c_{i} c_{j}^{\star}}{\hbar^{2}} \mathcal{R} \frac{1-e^{-i \omega_{i j} t}}{i \omega_{i j}}
$$

Linear population growth is seen similar to the growth seen in collisionally broadened CW case. Each excited state is pumped at an equal rate (assuming that the dipole matrix elements of the excited states are the same). The more intense the field the larger the coherences between excited states. However, this comes with the caveat that populations are also pumped at a faster rate. Using these two equations C2 C3 the mixed state measure, $\mathcal{C}$ for white noise irradiated $V$ level system is:

$$
\mathcal{C}=\frac{\left|1-e^{-i \omega_{i j} t}\right|}{2 \omega_{i j} t}
$$

This equation assumes that the transitions between the ground state to the excited states have the same dipole moment. $\mathcal{C}$ is then plotted in Fig. 14:

From Fig. 14, it is clear that after several excited state periods, the coherences of the system becomes a small fraction of population, thus approaching a mixed state. It should also be noted that this excited state coherence fraction is independent of pump power, $\mathcal{R}$. This means that there is no way to enhance the excited state coherence by simply turning up the power of the source.

The collisionally broadened CW source (Eq. (1)) cannot readily be related to white noise. It would be natural to assume that in the limit $\tau_{d} \rightarrow 0$ the collisionally broadened CW source should converge to white noise, but this is not the case. In that limit, $\mathcal{U} \rightarrow 0$ and thus the frequency spectrum of the laser also approaches zero. White noise, on the other hand, has a uniform frequency spectrum. 


\begin{tabular}{|l|l|}
\hline Parameter & Value \\
\hline$D B V^{+}$Frequency & $529 \mathrm{THz}$ \\
\hline$D B V^{-}$Frequency & $510 \mathrm{THz}$ \\
\hline$\tau_{d}$ & $1.32 \mathrm{fs}$ \\
\hline$\omega_{0}$ Frequency & $519.5 \mathrm{THz}$ \\
\hline$\mu$ & $12.8 \mathrm{Debye}$ \\
\hline Solar Flux & 130,000 lux \\
\hline
\end{tabular}

TABLE I: Parameters used in perturbative calculations of PC645.

\section{Appendix D: Parameters Used in PC645 Calculation}

The parameters used in the perturbative calculation of the incoherent irradiation of PC645 are listed in Table If. The values for the $D B V^{ \pm}$states as well as dipole moment were taken from the literature [21]. The dipole moment of the two DBV c,d sites were averaged and used for as the dipole moment of the $D B V^{ \pm}$states. This is valid as long as there is no dipole matrix element between the DBVc,d states, i.e. $\langle D B V c|\mu| D B V d\rangle=0$. Values of the coherence time of the radiation was taken from the approach of Wolf [14] and the solar flux was taken from values of the solar flux in midday [22]. 
[1] E. Collini, C. Y. Wong, K. E.Wilk, P. M. G. Curmi, P. Brumer and G. D. Scholes, Nature 463, 644 (2010).

[2] G. S. Engel, T. R. Calhoun, E. L. Read, T.-K. Ahn, T. Mancal, Y.-C. Cheng, R. E. Blankenship and G. R. Fleming, Nature 446, 782 (2007).

[3] X.-P. Jiang and P. Brumer, J. Chem. Phys. 94, 5833 (1991).

[4] P. Brumer and M. Shapiro, Proc. Natl. Acad. Sci. USA 109, 19575 (2012)

[5] T. Mancal and L. Valkunas, New J. Phys. 12, 065044 (2010).

[6] L. A. Pachon and P. Brumer, Phys. Rev. A 87, 022106 (2013).

[7] A. Han, M. Shapiro and P. Brumer, J. Phys. Chem. A 117, 8199 (2013).

[8] A. Aharony, S. Gurvitz, O. Entin-Wohlman and S. Dattagupta, Phys. Rev. B 82, 245417, (2010).

[9] V. V. Kozlov, Y. Rostovtsev and M. O. Scully, Phys. Rev. A 74, 063829 (2006).

[10] M. Fleischhauer, C. H. Keitel and M. O. Scully, Opt. Commun. 87, 109 (1992).

[11] Y. Elran and P. Brumer, J. Chem. Phys. 121, 2673 (2004)

[12] Y. Elran and P, Brumer, J. Chem. Phys. 138, 234308 (2013)

[13] R. Loudon, The Quantum Theory of Light, Oxford University Press, Oxford (1983).

[14] C. L. Mehta and E. Wolf, Phys. Rev. 134, A1143 (1964).

[15] L. Mandel and E. Wolf, Optical Coherence and Quantum Optics, Cambridge University Press, New York (1995).

[16] M. Shapiro, P. Brumer, Principles of the Quantum Control of Molecular Processes, Wiley, New York (2003); M. Shapiro and P. Brumer, Quantum Control of Molecular Processes, 2nd edn, Wiley-VCH, Weinheim (2012).

[17] C.W. Gardiner, Handbook of Stochastic Methods for Physics, Chemistry and Natural Sciences, Springer Verlag, Berlin (1983). Chapter IV, page 257.

[18] Y-C Cheng, G.R. Fleming, Annu. Rev. Phys. Chem. 60, 241 (2009).

[19] L. A. Pachon, L. Yu and P. Brumer, Faraday Disc. 163, 485 (2013); L. A. Pachon and P. Brumer, J. Chem. 139, 164123 (2013).

[20] G. D. Scholes, G. R. Fleming, A. Olaya-Castro and R. van Grondelle, Nature 3, 763 (2011).

[21] A. B. Doust, K. E. Wilk, P. M. G. Curmi and G. D. Scholes, J. Photochem. Photobio. A 184, 
$1(2006)$.

[22] P. Schlyter, Radiometry and Photometry in Astronomy, http://stjarnhimlen.se/comp/radfaq.html Stockholm (2009).

[23] T. Grinev and P. Brumer (manuscript in preparation) 


\section{FIGURES}

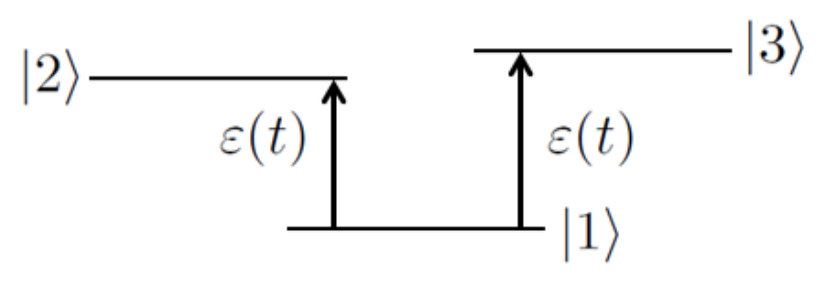

FIG. 1: Three level system used in our numerical scheme. Population is initially in the ground state $|1\rangle$ and is pumped to two non-degenerate excited states, $|2\rangle$ and $|3\rangle$. The frequency difference between the two excited states $\omega_{32}$ determines a characteristic excited state timescale $\tau_{c}=2 \pi / \omega_{32}$. 

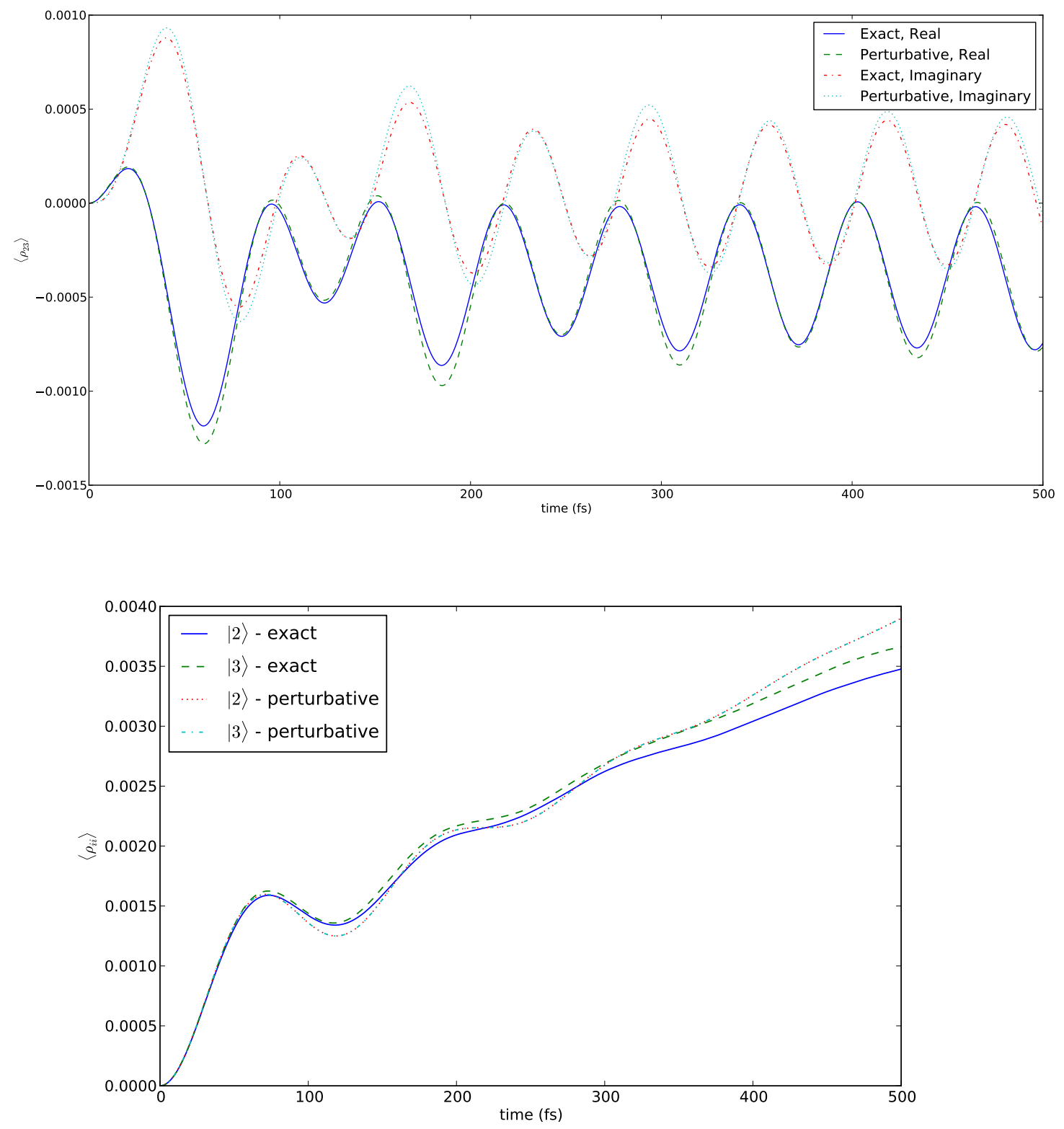

FIG. 2: (Top) Ensemble averaged excited state coherence $\left(\left\langle\rho_{23}\right\rangle\right)$ plotted versus perturbative coherences for a three level ladder system excited by thermally broadened CW source. (Bottom) Excited state populations for both perturbative and numerical results for a three level ladder system excited by thermally broadened CW source. $\tau_{c}=60$ fs and $\tau_{d}=120$ fs for both figures. 

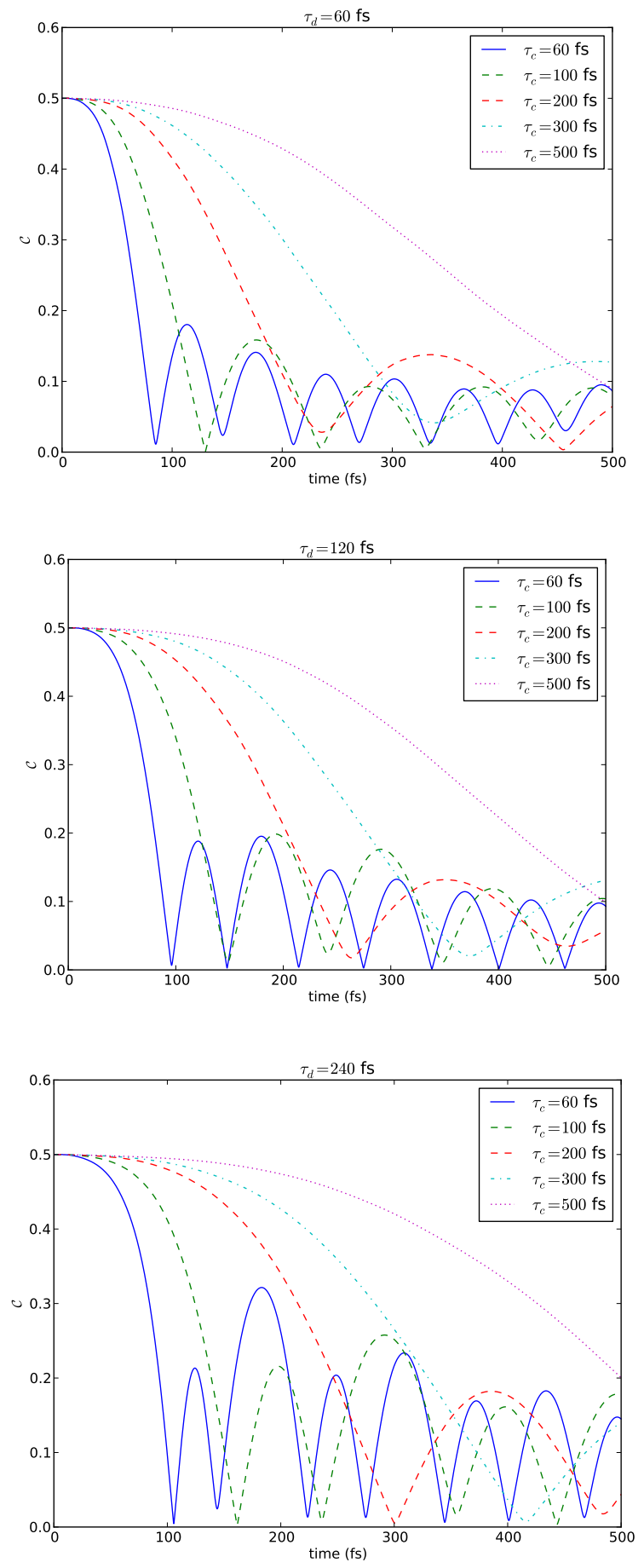

FIG. 3: $\mathcal{C}$ plotted against time for three level ladder system excited by thermally broadened CW source for various level splittings at fixed $\tau_{d}$. The values of $\tau_{d}$ used are $\tau_{d}=60 \mathrm{fs}$ (top), $\tau_{d}=120 \mathrm{fs}$ (middle) and $\tau_{d}=240 \mathrm{fs}$ (bottom). For large values of time, the quantity $\mathcal{C}$ becomes smaller and as $t \rightarrow \infty$ the value $\mathcal{C}$ approaches zero. 

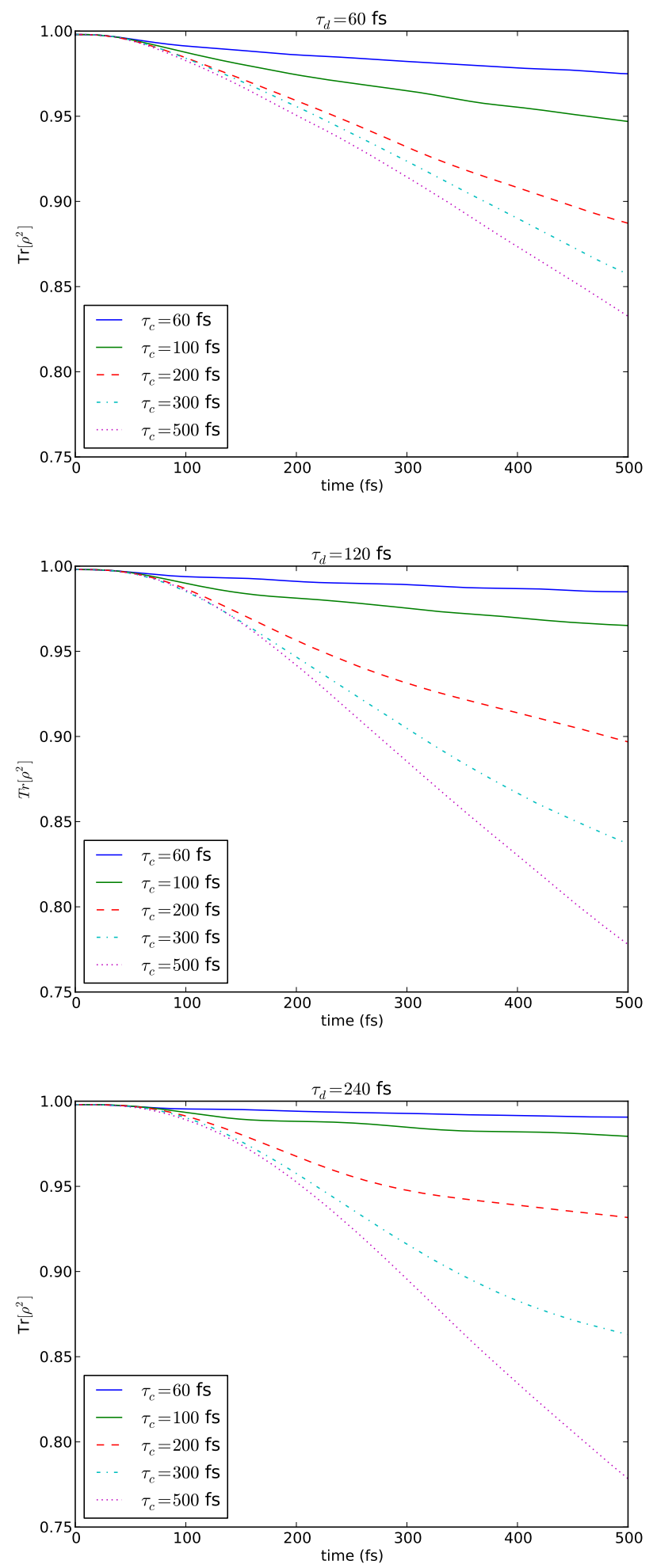

FIG. 4: System purity $\operatorname{Tr}\left(\rho^{2}\right)$ plotted as a function of time for various excited state periods $\tau_{c}$ and radiation coherence times $\tau_{d}$ for a three level ladder system excited by thermally broadened CW source. As the excited state period $\tau_{c}$ becomes larger, the purity of the system decreases at a faster rate. The following $\tau_{d}$ times are shown: $60 \mathrm{fs}$ (top), $120 \mathrm{fs}$ (middle) and $240 \mathrm{fs}$ (bottom). 

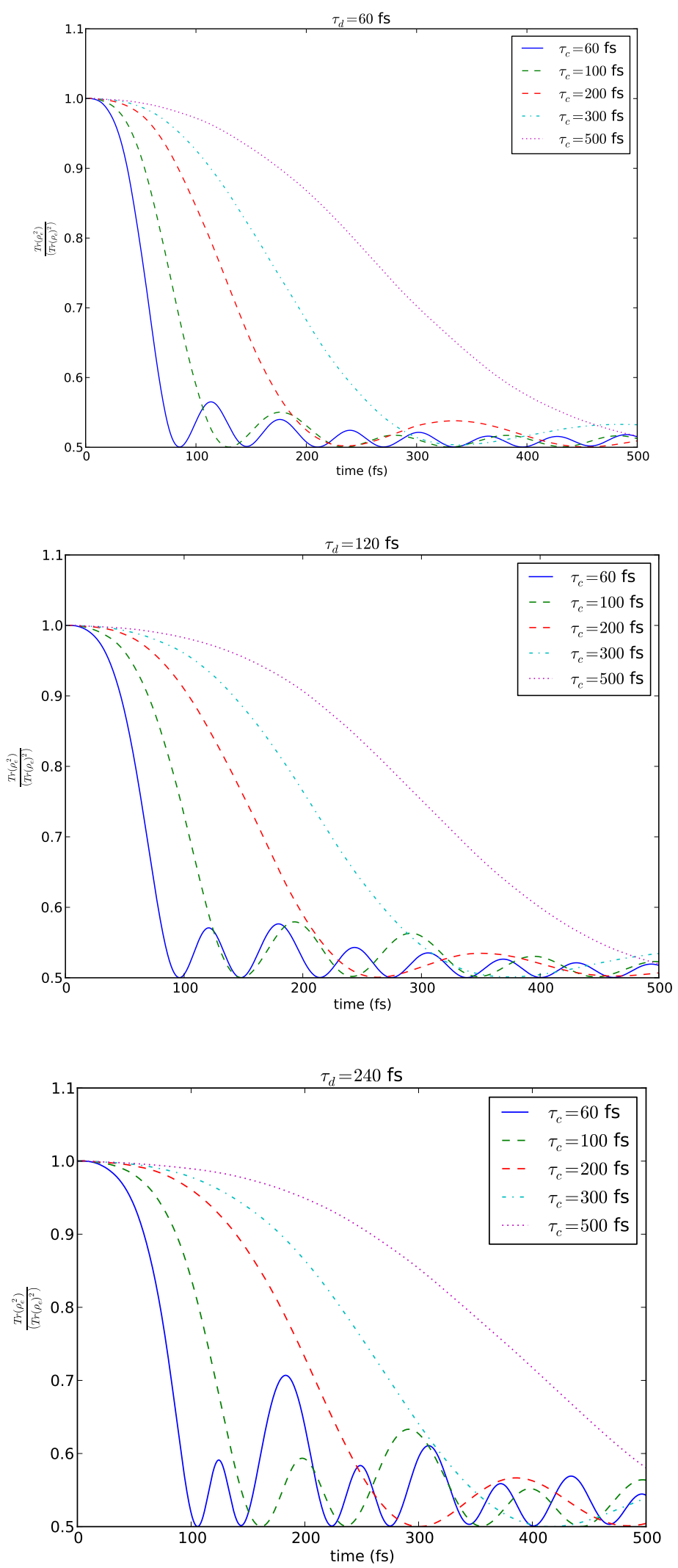

FIG. 5: Excited state purity plotted against time for three level ladder system excited by thermally broadened CW source for various level splittings, $\tau_{c}$ at various $\tau_{d}$. The following $\tau_{d}$ times are shown: $60 \mathrm{fs}$ (top), $120 \mathrm{fs}$ (middle) and $240 \mathrm{fs}$ (bottom). 


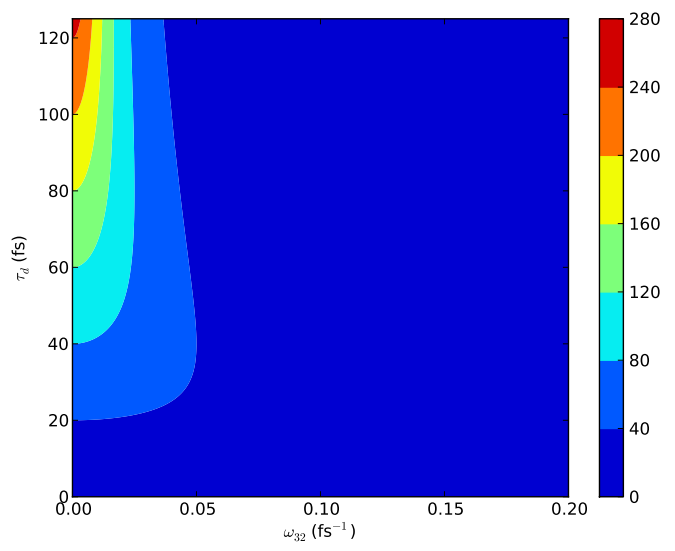

FIG. 6: Plot of $\mathcal{U}\left(\omega_{32}, \tau_{d}\right)$, red regions represent highest intensity and blue regions represent lowest intensity. 

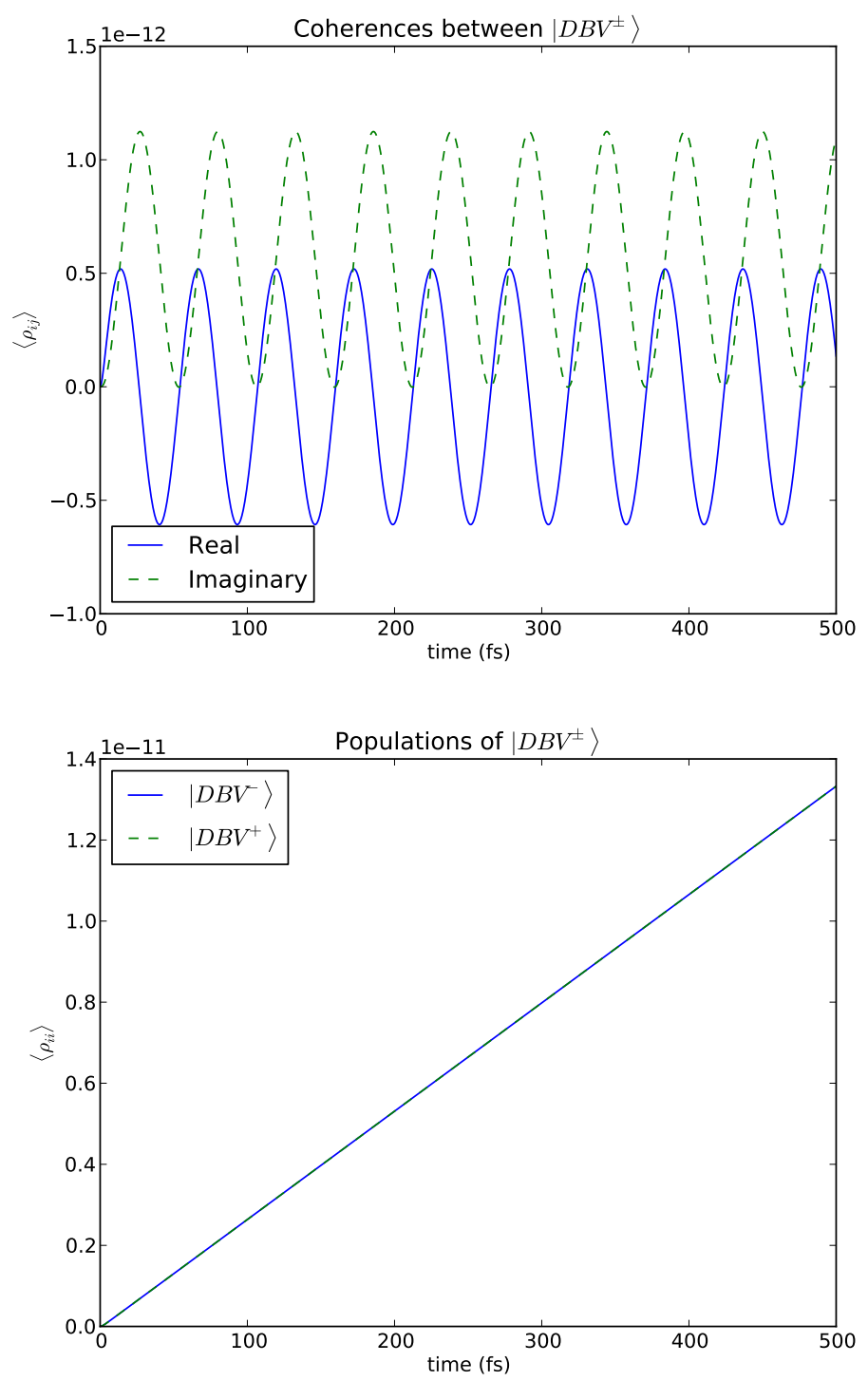

FIG. 7: (Top) Coherences between the $\left|D B V^{-}\right\rangle$and $\left|D B V^{+}\right\rangle$in the toy PC645 model as a function of time for the sudden turn-on case. (Bottom) Populations of $\left|D B V^{-}\right\rangle$and $\left|D B V^{+}\right\rangle$in the toy model of PC645 as a function of time for the sudden turn-on case. Excitation frequency of the laser is in the middle of the two transitions. 


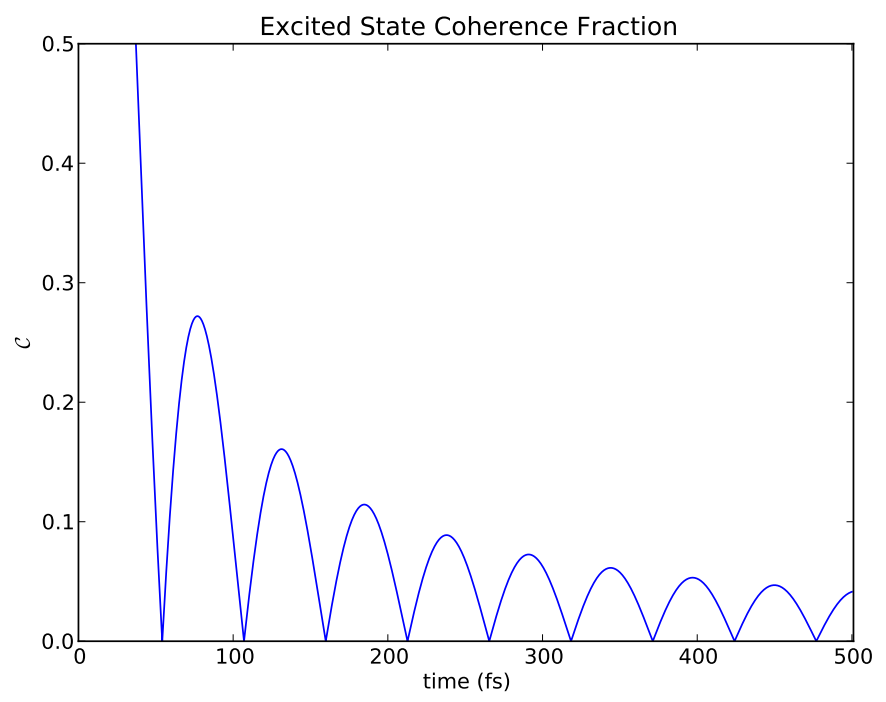

FIG. 8: Plot of $\mathcal{C} \equiv \frac{\left|\rho_{23}\right|}{\rho_{22}+\rho_{33}}$ for a toy model of PC645 upon excitation by a collisionally broadened CW source, for parameters indicated in the text.

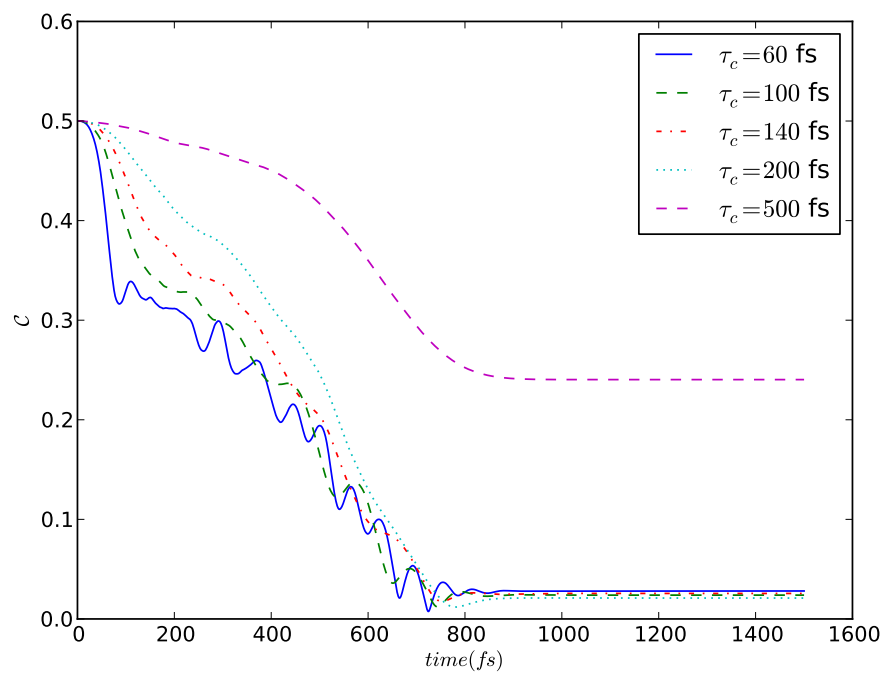

FIG. 9: Plot of $\mathcal{C}$ for various excited state splittings, $\tau_{c}$ for long incoherent pulses incident on a three level ladder system. The pulse used is $1 \mathrm{ps}$ in duration. $\tau_{d}=120 \mathrm{fs}$. The frequency center of the pulse is chosen to be in the center of the two transitions. 


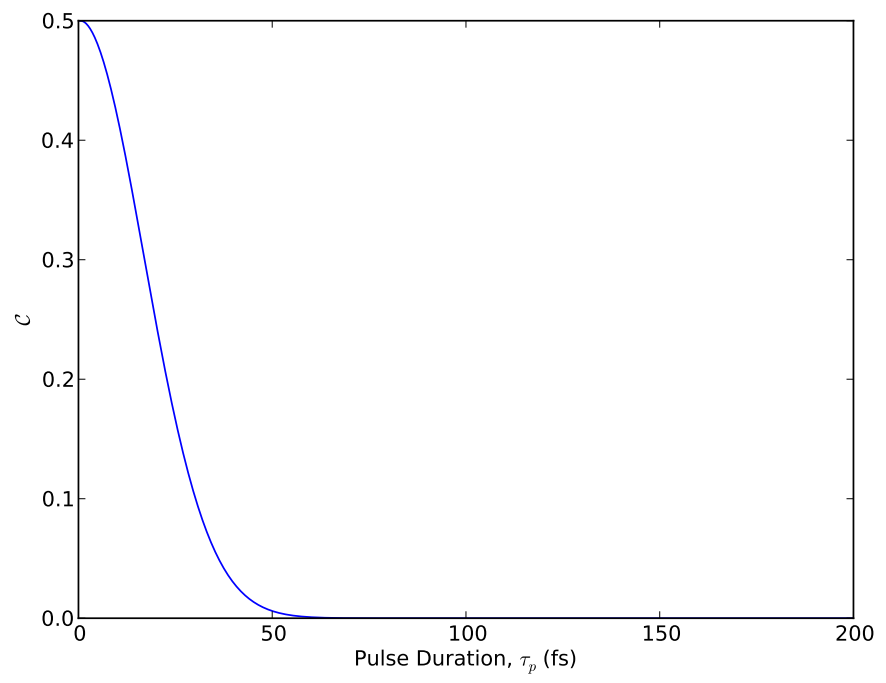

FIG. 10: $\mathcal{C}$, the magnitude of the post pulse excited state coherence fraction of a toy model of PC645, plotted as a function of pulse duration, $\tau_{p}$. For long pulses, it is evident that post pulse coherence is extremely small. 

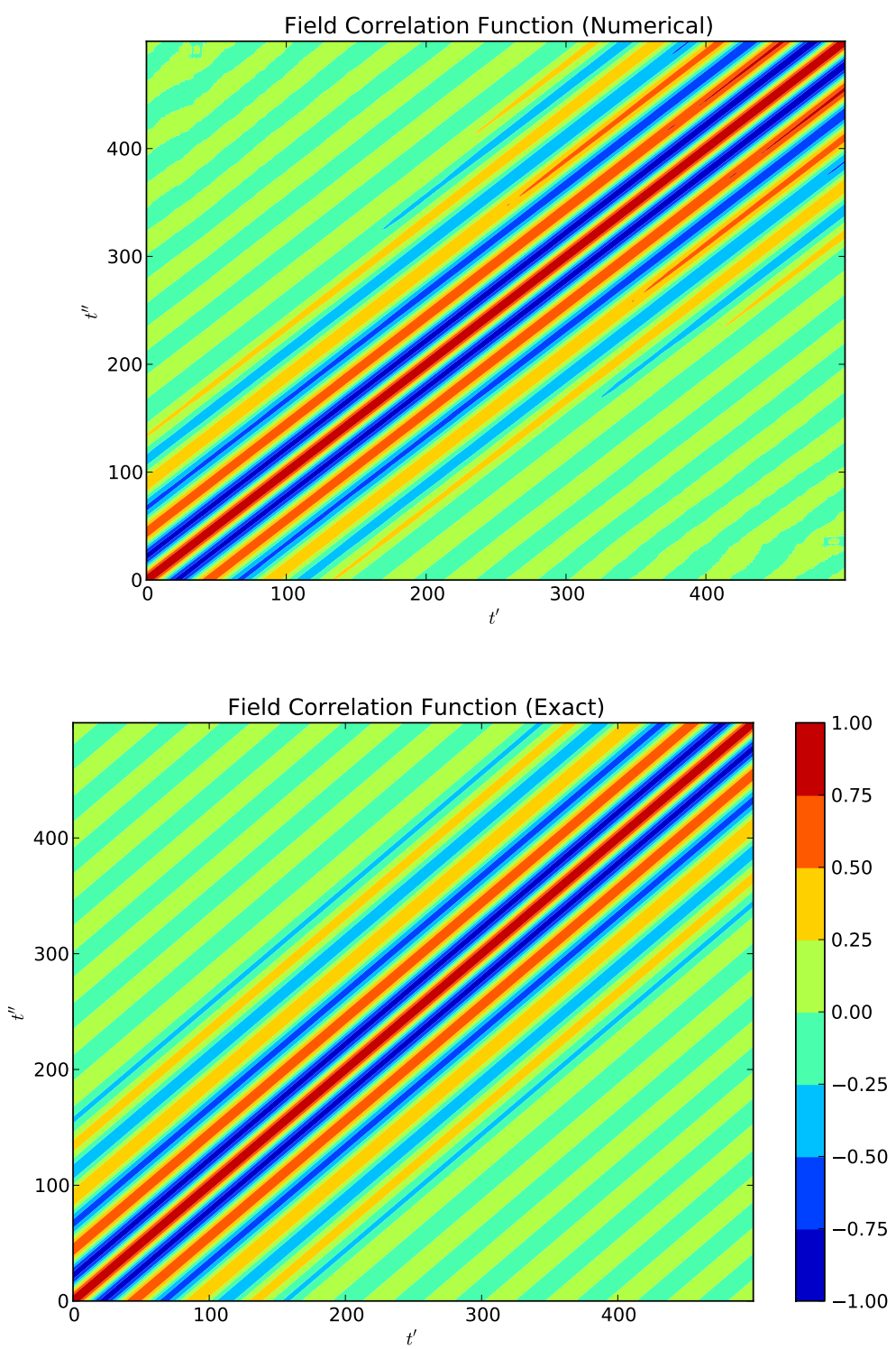

FIG. 11: Comparison of numerically generated (top) and numerical [Eq. (1)] (bottom) $\left\langle\varepsilon\left(t^{\prime}\right) \varepsilon\left(t^{\prime \prime}\right)\right\rangle$. Both plots share the same color legend. The times are in units of femtosecond. 

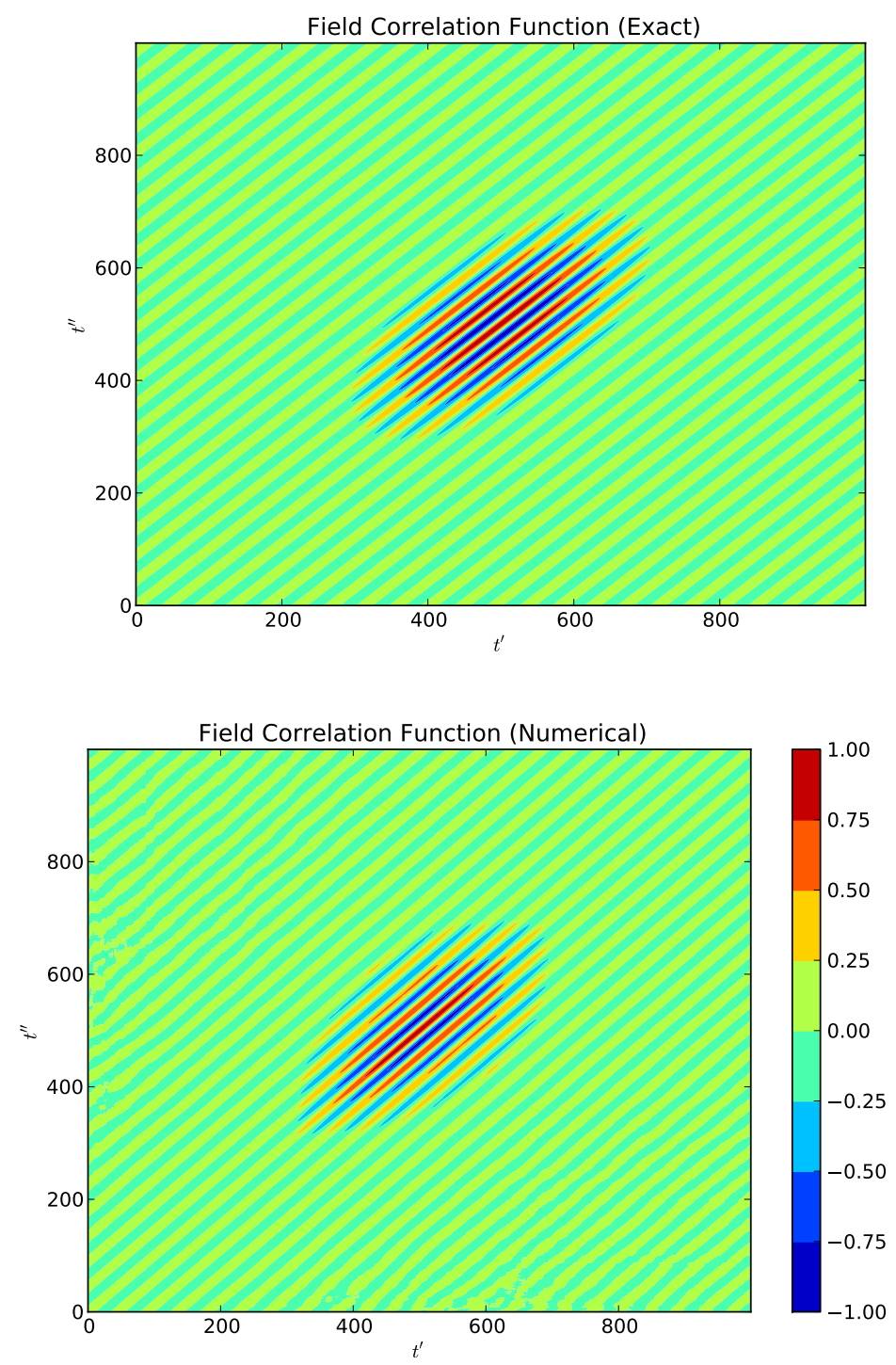

FIG. 12: (Top) Exact contour plot of the correlation function in Eq. 2 and (Bottom) numerical reproduction of correlation for the noisy pulsed source. Both plots share the same color legend. The times are in units of femtosecond. 


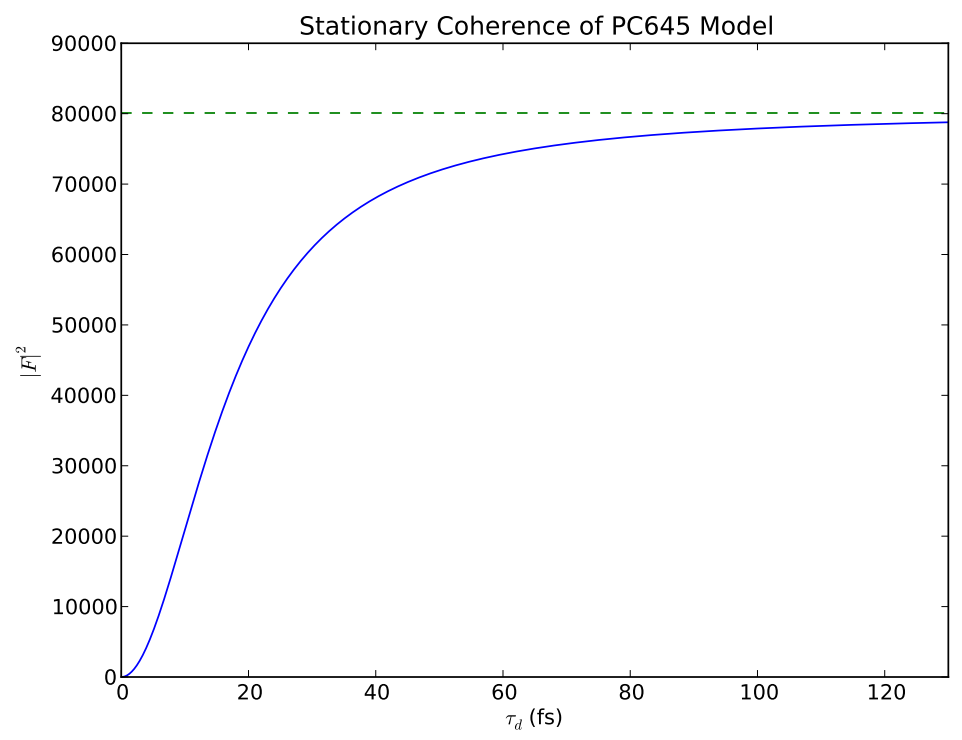

FIG. 13: Plot of $|F|^{2}$ using the parameters of PC645. Solid line is the stationary coherence as a function of $\tau_{d}$ while the dashed line represents the saturation level from Eq. (B10).

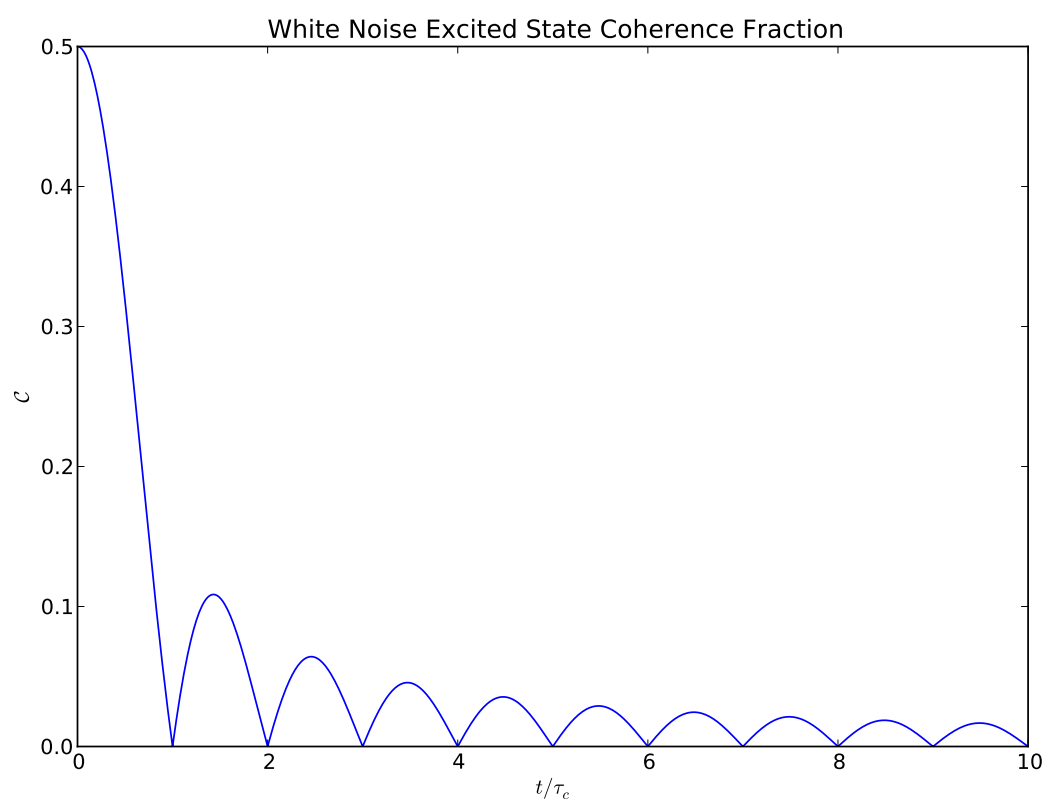

FIG. 14: Plot of $\mathcal{C}=\frac{\left|\rho_{23}\right|}{\rho_{33}+\rho_{22}}$ for a three level system irradiated with white noise as a function of dimensionless time. $\tau_{c}=\frac{2 \pi}{\omega_{i j}}$ 